\title{
Prevalence and risk factors for myopia among school children in Aba, Nigeria
}

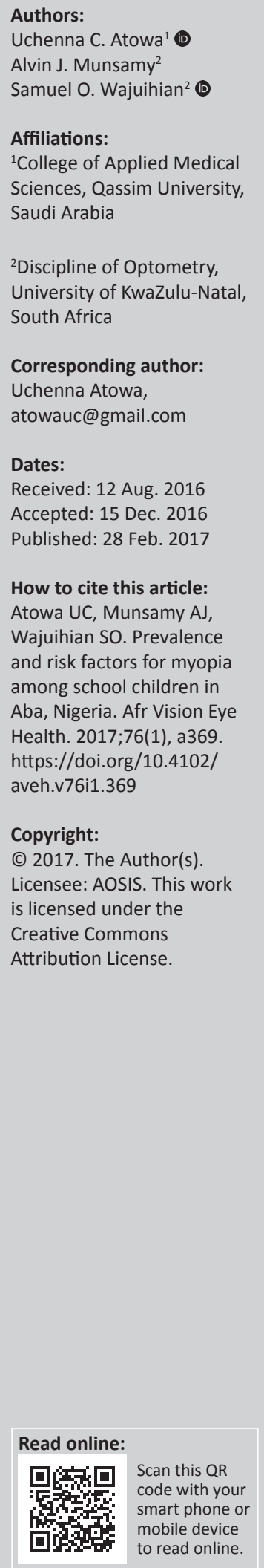

\begin{abstract}
Aim: To study the prevalence of myopia among school children in Aba, Nigeria.
Methods: This cross-sectional study was conducted in public and private (primary and secondary) schools. A multi-stage random sampling technique was used for selecting participants aged between 8 and 15 years from 12 schools in Aba, Nigeria. Data were analysed for 1197 children who underwent a comprehensive eye examination. The children were divided according to the following criteria: age groups (group 1 [8-11 years] or group 2 [12-15 years]), gender (male or female), level of education (primary or secondary) and type of school (public or private). Myopia was defined as spherical equivalent refraction (SER) $\leq-0.50 \mathrm{D}$ in the poorer eye.

Results: The prevalence of myopia was estimated to be $2.7 \%$. Of the 96 children with refractive error, $78.1 \%$ were uncorrected. In using logistic regression analysis, risk of developing myopia was associated with older age groups (odds ratio [OR]: 1.20; 95\% confidence interval [CI], 0.16-9.11; $p<0.010)$ and higher level of education (OR: 1.73; 95\% CI, $1.05-2.86 ; p<0.030)$. There was no significant difference in myopia prevalence between male and female children $(p=0.89)$.

Conclusion: Although the prevalence of myopia and overall prevalence of refractive error in school children in Aba were low, the high prevalence of uncorrected refractive error is a significant public health problem. An effective and sustainable children's vision screening programme is needed to prevent visual impairment and blindness.
\end{abstract}

\section{Introduction}

Recent epidemiological studies ${ }^{1,2,3,4,5,6,7,8}$ across the world have provided data on the prevalence and progression of myopia in children. In particular, studies in China, Singapore and Taiwan highlight the relatively high prevalence of myopia in Asian children, especially in urban areas. Among the 12-year-old children, the prevalence of myopia is $64.9 \%$ in China, ${ }^{1} 62.0 \%$ in Singapore ${ }^{2}$ and $56.0 \%$ in Taiwan $^{3}$ in comparison with $20.0 \%$ in the United States, ${ }^{4} 10.9 \%$ in Australia, ${ }^{5} 9.7 \%$ in urban India ${ }^{6,7}$ and $19.2 \%$ in Vietnam. ${ }^{8}$ In Africa, children showed a lower prevalence of myopia when compared with other regions of the world. Studies in Ghana, ${ }^{9}$ Ethiopia, ${ }_{10}^{10}$ Uganda, ${ }^{11}$ South Africa ${ }^{12}$ and Nigeria ${ }^{13}$ reported a myopia prevalence of $1.7 \%, 2.6 \%, 4.4 \%, 4.0 \%$ and $4.4 \%$, respectively. Overall, $80 \%-100 \%$ of the total refractive errors observed in African children were uncorrected. ${ }^{10,11,12,13}$

The Refractive Error Study in Children (RESC) surveys $8,12,14,15,16$ conducted in various countries identified the increasing prevalence of myopia and harmful effects of even a modest amount of uncorrected myopia on vision as a significant public health problem. Besides having to hold books very close and sit in front of the classroom to be able to see adequately, a child with myopia may develop sight-threatening complications and permanent visual impairment that may affect the child's social, educational and psychological development. ${ }^{17,18,19,20}$ In addition, direct and indirect costs related to myopia exert enormous socio-economic burden on the society.

Despite the consequences of uncorrected refractive error in children, there are no national data on prevalence of refractive error among children in Nigeria. ${ }^{21,22}$ Although, a few studies exist for some cities across the country, ${ }^{13,22,23}$ to the best of our knowledge, no RESC survey has been reported on the prevalence of myopia in school children in Aba, Nigeria. This study intends to provide information on the prevalence of myopia among school children in Aba, which will be necessary for effective and efficient eye health planning and education. 


\section{Methods Study design}

This was a cross-sectional school-based study conducted in Aba, a commercial city in Abia State, Nigeria. Aba comprises two local government areas, namely, Aba South and Aba North, with a total population of 534265 according to 2006 national census. ${ }^{24}$ Primary and secondary education is served by public and private institutions. Eyecare services are also provided by private and public health facilities. The eyecare services in the public sector are restricted to one primary and one tertiary health centre in the whole city of Aba. Private sector eye clinics are primarily located in the commercial city centres and operate on a commercial basis.

The study population included school children aged 8-15 years attending primary and secondary schools in Aba. Both private and public schools were included in the study and all schools follow the same national educational system and curriculum.

\section{Sample selection}

The study participants were selected through multi-stage random sampling. The city of Aba was divided into three areas considering the population and socio-economic characteristics of the areas. The sampling design included stratification at public and private schools. Four schools (two public primary and secondary schools and two private primary and secondary schools) were randomly selected from each of the three areas. Overall, 12 schools were selected out of 85 schools in the area. The next stage was the recruitment of children aged 8-15 years from grades 3-11 in each of the participating schools by systematic random sampling. Grades 3-6 included those in primary schools while grades 7-11 were those in secondary schools. One to three classes were randomly selected from each level with a minimum cluster size of 25 students. All the students within a class were invited to participate. If the minimum sample of 25 was not achieved from the first class, students from the second selected class were used to attain the required sample size. Every second or third child starting from the first child in a class register was included until the desired cluster sample size was reached.

The sample size was calculated using the formula: $N=(\mathrm{Z})^{2}(1.0-p)(p) /(\mathrm{b})^{2}$, where $\mathrm{N}$ is the minimum sample size, $p$ is the anticipated prevalence (assumed to be 50\%), $\mathrm{b}$ is the desired error bound taken as $5 \%$ and $Z=1.96$ for a $95 \%$ confidence interval (CI). A design effect of 2.0 and $10 \%$ contingency factor to account for attrition was also considered. The total number of participants was 1261 .

\section{Study procedure}

Selected schools were visited individually in advance to seek permission and cooperation of the school authorities. An invitation to participate in the study was sent to parents or guardians of the children recruited for the study together with the consent form and information leaflet. A parents-teachers association (PTA) meeting was held prior to the survey, where the details of the study were clearly restated. Children whose parents gave signed consent for an examination under full cycloplegia were included in the study.

The vision assessment was based on the children RESC protocol $^{26}$ with specific modifications to serve the purpose of this study as well as the availability and affordability of instruments. Examinations were performed by a team that comprised three optometrists, two ophthalmic nurses and two clinical assistants. The study team was trained before the survey fieldwork and was familiarised with the study procedure. A pilot field exercise to validate the data collection instruments was organised at a primary and a secondary school not included in the actual survey.

Distance visual acuity was measured monocularly and binocularly with a retro-illuminated logMAR chart (Precision Vision, Villa Park, IL, USA) containing five optotypes at a $4 \mathrm{~m}$ testing distance from the chart. Anterior and posterior segments examination, as well as ocular motility assessment and pupil evaluation, was performed with a Welch Allyn diagnostic set. Ocular alignment was initially assessed by the corneal reflex (Hirschberg) test, and then followed by the cover test at distance and near.

Cycloplegia was achieved by giving two drops of cyclopentolate (1\%) at five-minute intervals. Cycloplegia was considered full when the pupil was fixed and $\geq 6 \mathrm{~mm}$ in diameter. If on evaluation after 20 minutes, the pupillary light reflex was still present, a third drop was administered. Cycloplegic autorefraction was carried out with the Topcon RM-8000B (Topcon Corporation, Tokyo, Japan) autorefractometer 60 minutes after first instillation of the drops. An average of three readings was taken for each child, and all participants underwent a cycloplegic refraction. ${ }^{12,27}$

\section{Definition}

Refractive error (RE) was classified based on the spherical equivalent refraction (SER) calculated as the sum of the sphere and half the cylindrical component. Myopia was defined as SER $\leq-0.50 D^{5,12}$ in the poorer eye and was sub-classified as low $($ SER $<-0.50 \mathrm{D}>-3.00 \mathrm{D})$, medium $(\mathrm{SER}<-3.00 \mathrm{D}>-6.00 \mathrm{D})$ and high (SER $<-6.00 \mathrm{D})$. Hyperopia was defined as SER $\geq+$ $2.00 \mathrm{D}$ in the poorer eye and was sub-classified as low $(\mathrm{SER} \geq+2.00 \mathrm{D}<+4.00 \mathrm{D})$, medium $(\mathrm{SER} \geq+4.00 \mathrm{D}<+6.00 \mathrm{D})$ and high (SER $\geq+6.00 \mathrm{D})$. Astigmatism was defined as cylinder (cyl.) $\geq 0.75$ D. ${ }^{5}$

\section{Data analysis}

Data entry was performed on a daily basis, alongside the daily fieldwork. Additional data review and consistency checks were conducted once data collection and entry were completed for an entire school or cluster by an experienced 
data officer. The statistical analysis was performed using a commercially available Statistical Package for Social Science software (SPSS for Windows, Version 20.0, IBM-SPSS, Chicago, IL, USA).

The $z$-test for two populations was used to compare proportions. Logistic regression was used to compare the prevalence of myopia between age groups, gender, level of education (secondary versus primary) and type of school (private vs. public school). Odds ratios (OR) and 95\% CIs were presented. A $p$ value $<0.05$ was used as the criterion for statistical significance.

\section{Ethical considerations}

Approval for the study was obtained from College of Medicine Research Ethics Committee, University of Nigeria. Informed written consent and assent were obtained from the parents and children, respectively, before examination after adequate information about the study had been provided to them. The study followed the tenets of the Declaration of Helsinki for research involving human subjects. ${ }^{25}$

\section{Results}

\section{Study characteristics}

The characteristics of the study sample are summarised in Table 1. Of 1261 school children randomly recruited from 12 schools in Aba, 1212 (96.1\%) participated in the study. However, only 1197 (94.9\%) children with complete relevant data were included for analysis. The overall mean age of the participants was $11.5 \pm 2.3$ (s.d.), range 8-15 years. All the participants were divided into two age groups: group 1 (8-11 years) with mean age of $9.5 \pm 1.2$ and group 2 (12-15 years) with mean age of $13.5 \pm 1.1$. Of the participants, $659(55.1 \%)$ were female, while $538(44.9 \%)$ were male; $581(48.5 \%)$ children were from public schools, whereas $616(51.5 \%)$ were from private schools; 549 (45.9\%) were from primary schools and 648 (54.1\%) were from secondary schools.

TABLE 1: Characteristics of the study population.

\begin{tabular}{lcc}
\hline Characteristics & Number of participants $(\boldsymbol{N})$ & Percentage \\
\hline All children & 1261 & 100.0 \\
$\quad$ Participants & 1212 & 96.1 \\
Participants with complete data & 1197 & 94.1 \\
Age & & \\
$\quad$ Group 1 (8-11), mean age $(9.5 \pm 1.2)$ & 595 & 49.7 \\
$\quad$ Group 2 (12-15), mean age (13.5 \pm 1.1$)$ & 602 & 50.3 \\
Gender & & \\
Female & 659 & 55.0 \\
$\quad$ Male & 538 & 45.0 \\
Type of school & & 48.5 \\
$\quad$ Public & 581 & 51.5 \\
$\quad$ Private & 616 & \\
Level of education & & 45.9 \\
$\quad$ Primary (grades 3-6) & 549 & 54.1 \\
\hline Secondary (grades 7-11) & 648 & \\
\hline
\end{tabular}

\section{Refractive error}

The prevalence of RE was analysed based on cycloplegic autorefraction readings. The prevalence of RE consisted of $0.9 \%$ hyperopia, $2.7 \%$ myopia and $4.4 \%$ astigmatism. Among the myopes $(2.7 \%)$, low, medium and high accounted for $87.5 \%, 9.4 \%$ and $3.1 \%$, respectively. The mean SER for myopia was $-1.48 \mathrm{D} \pm 2.12 \mathrm{D}$ in the poorer eye. The mean SER of myopia was $-1.30 \mathrm{D} \pm 0.90 .63 \mathrm{D}$ and $-1.64 \mathrm{D} \pm 2.83 \mathrm{D}$ for boys and girls, respectively. All hyperopia recorded based on the definition criteria for the study was of low degree with a mean SER of $+2.81 \mathrm{D} \pm 0.63 \mathrm{D}$ in the poorer eye.

There was a significant difference in myopia prevalence within the age groups $(p=0.001)$ (Table 2). Myopia prevalence was $8(1.3 \%)$ in age group 1 and $26(4.3 \%)$ in age group 2 . Gender prevalence shows that although myopia was marginally higher in female participants [18 (2.7\%)] than in male participants $[14(2.6 \%)]$, the difference was not statistically significant ( $z$-test for two proportions, $p=0.890$ ). The prevalence of myopia was significantly higher in secondary schools $27(4.1 \%)$ than in primary schools $5(0.9 \%)$ ( $z$-test for two proportions, $p=0.001$ ). The number of myopic children observed in the study comprised $9(1.5 \%)$ public school children and $23(3.7 \%)$ private school children. The difference was statistically significant ( $z$-test for two proportions, $p=0.020$ ) (Table 2).

In the multivariate model, the presence and absence of myopia was the dependent factor, while the variables that were significantly associated with myopia in univariate analysis were the independent factors. After adjusting for age, it was found that myopia remained significantly associated with older age groups (OR: 1.20; 95\% CI, $0.16-9.11 ; p<0.010)$ and higher grades (OR: 1.73; 95\% CI, $1.05-2.86 ; p<0.030$ ). Type of school (private vs. public) was no longer associated with myopia $(p=0.650)$.

Of 96 children who required RE correction in one or both eyes, only $21(21.9 \%)$ presented with spectacle corrections

TABLE 2: Prevalence of myopia by age group, gender, level of education and type of school.

\begin{tabular}{lcccc}
\hline Categories & Total screened $(N)$ & \multicolumn{2}{c}{ Myopia (n-32) } & $p$ \\
\cline { 3 - 4 } & & $n$ & $\%$ & \\
\hline Age group & 595 & 8 & 1.3 & $0.001^{*}$ \\
$\quad$ Group 1 & 602 & 26 & 4.3 & \\
$\quad$ Group 2 & & & & 0.89 \\
Gender & 595 & 18 & 2.7 & \\
$\quad$ Female & 538 & 14 & 2.6 & \\
$\quad$ Male & & & & $0.001^{*}$ \\
Level of education & 549 & 5 & 0.9 & \\
$\quad$ Primary & 648 & 27 & 4.1 & \\
$\quad$ Secondary & & & & $0.02^{*}$ \\
Type of school & 581 & 9 & 1.7 & \\
$\quad$ Public & 616 & 3 & 3.5 & \\
$\quad$ Private & 1197 & 32 & $\mathbf{2 . 7}$ & - \\
\hline Total & & & & \\
\hline
\end{tabular}

*, Statistically significant $p$ values. 
TABLE 3: Refractive error and spectacle use by age group, gender, type of school and level of education.

\begin{tabular}{|c|c|c|c|c|c|}
\hline \multirow[t]{2}{*}{ Categories } & \multirow[t]{2}{*}{ Total RE $(n=96)$} & \multicolumn{2}{|c|}{$\begin{array}{l}\text { Children with } \\
\text { spectacles }\end{array}$} & \multicolumn{2}{|c|}{$\begin{array}{l}\text { Children without } \\
\text { spectacles }\end{array}$} \\
\hline & & $n$ & $\%$ & $n$ & $\%$ \\
\hline \multicolumn{6}{|l|}{ Age group } \\
\hline Group 1 & 34 & 7 & 20.6 & 27 & 79.4 \\
\hline Group 2 & 62 & 2 & 22.6 & 48 & 77.4 \\
\hline \multicolumn{6}{|l|}{ Gender } \\
\hline Male & 42 & 9 & 21.4 & 33 & 78.6 \\
\hline Female & 55 & 12 & 21.8 & 43 & 78.2 \\
\hline \multicolumn{6}{|c|}{ Type of school type } \\
\hline Public & 37 & 6 & 16.2 & 31 & 83.8 \\
\hline Private & 59 & 15 & 25.4 & 44 & 74.6 \\
\hline \multicolumn{6}{|c|}{ Level of education } \\
\hline Primary & 36 & 4 & 11.1 & 32 & 88.9 \\
\hline Secondary & 60 & 17 & 28.3 & 43 & 71.7 \\
\hline
\end{tabular}

$\mathrm{RE}$, Refractive error.

including six children who were under corrected and had normal vision with refraction (Table 3). Of the children, 33\% without spectacles during the eye examination were myopic.

\section{Discussion}

This study aimed to access the prevalence of myopia among school children aged 8-15 years in Aba, Nigeria. An overall prevalence of RE of $8.0 \%$ was observed, which is comparable to that reported in the cosmopolitan city of Ikeja Lagos, Nigeria ${ }^{23}$ but lower than the prevalence of RE observed in other geographic locations such as Qassim in Saudi Arabia $(18.6 \%),{ }^{27}$ Vung Tau in Vietnam $(21.6 \%)^{8}$ and Sydney in Australia $(25.4 \%){ }^{5}$

The prevalence of myopia was $2.7 \%$ in school children in Aba. This estimate is also comparable with the prevalence reported in Nigerian school children and other African countries. ${ }^{9,12,13}$ For instance, in Birnin Kebbi, Nigeria, ${ }_{1}^{13}$ it was $2.9 \%$; in Durban, South Africa, ${ }^{12}$ the prevalence was $2.9 \%$; and in Swedru, Ghana, ${ }^{9}$ it was $1.7 \%$. However, these were lower than those reported in China $(64.4 \%),{ }^{1}$ Vietnam $(20.4 \%)^{8}$ and Australia $(6.5 \%) .{ }^{5}$ The difference can be partly attributed to older age groups and sample size because myopia increases with age. It can also be attributed to genetic susceptibility to myopia that varies across different ethnic origins and cultural settings. ${ }^{8,19,20}$

Consistent with previous studies, $8,12,14,15,16,27$ an increased prevalence of myopia with older school children was observed with a corresponding increase in the level of education. The findings from numerous RESC studies ${ }^{8,14,15,16,27}$ in various countries revealed that depending on schooling and learning system, early significant hyperopia decreases rapidly from age 5 to insignificant levels by the age of 15 , with a noticeable myopic shift taking place around the age of 12 when secondary school begins. Similarly, our study shows that myopia was four times higher between the ages of 12 and 15 years (age group 2) corresponding to the age of the children in secondary school. Intensive near work over time could result in retinal defocus which leads to axial length elongation, thereby causing myopia ${ }^{8,18,19,20}$. Thus, the age increase in myopia prevalence corresponding with school year may be attributed to more hours of near work and indoor activities.

There was no significant difference in the prevalence of myopia between male and female participants in our study. This finding agrees with those of several studies on African children ${ }^{12,15,23,27}$ and is comparable with the results of a study in Nepal. ${ }^{16}$ However, studies in Saudi Arabia ${ }^{27}$ and Finland ${ }^{28}$ found that the prevalence of myopia was significantly higher in female than in male subjects. These two studies included participants with active growth. As previous studies have associated early peak height velocity ${ }^{29}$ as well as intensive education ${ }^{8,14,15,16}$ with prevalence and progression of myopia in children, the authors suggest that the difference may have been influenced by the earlier growth and maturation rate of girls than that of boys in their study samples. In contrast, a study on childhood myopia (with subjects between 6 and 15 years) by Goss ${ }^{30}$ observed that gender has less effect on the prevalence of childhood myopia, and this agrees with our study. Similarly, Paudel et al. ${ }^{8}$ found no significant difference in myopia prevalence between male and female participants in their study sample; however, the authors observed that risk of developing myopia, such as indoor reading and less outdoor activities, was higher in girls than in boys.

It is possible that gender pathogenesis of refractive error is relatively significant in one ethnic origin than another, owing to cultural variations to lifestyle characteristics such as the number of hours spent on near work and outdoor activities between both sexes. ${ }^{8}$ Secondly, population age of the study samples, definition criteria for myopia and methods applied in data collection may account for the discrepancies in the results of these studies.

Uncorrected refractive error contributed to $78.1 \%$ of the total refractive error in school children in Aba (Table 3). Balarabe et al. ${ }^{13}$ observed the worst situation in Benin Kebbi, Nigeria, where $90 \%$ of uncorrected RE was responsible for the total RE in school children. These figures are significantly higher when compared to other geographic areas with efficiently established screening programmes and eye health education, such as Cairo in Egypt (57.4\%), ${ }^{31}$ Andhra Pradesh in India (61\%), ${ }^{6}$ and Yangxi $(60 \%)^{15}$ and Beijing $(56 \%)^{1}$ in China. The differences in spectacle coverage highlight the poor uptake of refractive services and spectacle needs among Nigerian school children, particularly in Aba. Some studies report that the low uptake of refractive services may be because of the unfounded belief about spectacle wear, caused by lack of awareness and ocular health education that parents believe that children using spectacles at an early age will suffer worsened vision and eventually become blind. ${ }^{9,23}$ Others report this may also be because of the inadequate manpower and disproportionate distribution of refractive services in communities including the high cost of eyecare services. ${ }^{13,21}$ The primary eyecare service is limited to one facility in the city of Aba, 
and refractive services are mainly provided by private eye clinics predominantly located in the commercial city centres. As a result, eyecare services are inaccessible and unaffordable to many people. Altogether, the data from this study warrant improvement in the effectiveness of eyecare programmes. Primary eyecare facilities in Aba may need to be increased for easier accessibility and wider coverage. Eye health service which will include ascertaining the visual status of children at first instance and subsequent periodic vision screening should be an integral part of the school health programme. In addition, we recommend the development of a standard screening protocol by eyecare practitioners that will target common visual problems in children which may lead to visual impairment and blindness.

\section{Conclusion}

The prevalence of myopia (2.7\%) and the overall prevalence of refractive error $(8.0 \%)$ from our study are low. However, the large proportion $(78.1 \%)$ of children with uncorrected refractive error is a significant public health concern. Even though uncorrected RE can be easily treated with spectacles, less than a quarter of children with RE were found using spectacles during examination. It is important to identify conditions affecting vision in a timely manner, as many common visual problems can be managed effectively once they are identified. The data from this study support the need for increased access to affordable child eye health, which will include routine vision screening in schools and ocular health education in the wider community.

\section{Acknowledgements}

We are grateful for the cooperation received from the management and staff of the schools that participated in the study. We also appreciate the support of Dr Ekpoma and Dr Chidi in the data collection.

\section{Competing interests}

The authors declare that they have no financial or personal relationships that may have inappropriately influenced them in writing this article.

\section{Authors' contributions}

U.C.A. was responsible for the study design, implementation and manuscript writing. A.J.M. and S.O.W. provided guidance on the study design, methodology manuscript writing and review.

\section{References}

1. You QS, Wu LJ, Duan JL, et al. Prevalence of myopia in school children in greater Beijing: The Beijing Childhood Eye Study. Acta Ophthalmol. 2014;92:e398-e406. https://doi.org/10.1111/aos.12299

2. Saw SM, Shankar A, Tan SB, et al. A cohort study of incident myopia in Singaporean children. Invest Ophthalmol Vis Sci. 2006;47:1839-1844. https://doi.org/10.1167/ iovs.05-1081

3. Lin LL, Shih YF, Tsai CB, et al. Epidemiologic study of ocular refraction among school children in Taiwan in 1995. Optom Vis Sci. 1999;76:275-281. https://doi. org/10.1097/00006324-199905000-00013
4. Zadnik K. The Glenn A. Fry award lecture. Myopia development in childhood. Optom Vis Sci. 1997;74:603-608. https://doi.org/10.1097/00006324-19970800000021

5. Jungham BM, Crewther SG. Prevalence of myopia among primary school children in eastern Sydney. Clin Exp Optom. 2003;86:339-345. https://doi.org/10.1111/ j.1444-0938.2003.tb03130.x

6. Dandona $\mathrm{R}$, Dandona L, Srinivas $\mathrm{M}$, et al. Refractive error in children in a rural population in India. Invest Ophthalmol Vis Sci. 2002;43:615-622.

7. Murthy GV, Gupta SK, Ellwein LB, et al. Refractive error in children in an urban population in New Delhi. Invest Ophthalmol Vis Sci. 2002;43:623-631.

8. Paudel $P$, Ramson $P$, Naduvilath, $T$, et al. Prevalence of vision impairment and refractive error in school children in Ba Ria-Vung Tau Province, Vietnam. Clin Exp Ophthalmol. 2013;42:217-226. https://doi.org/10.1111/ceo.12273

9. Ovenseri-Ogbomo GO, Assien R. Refractive error in school children in Agona Swedru, Ghana. S Afr Optom. 2010;69:86-92. https://doi.org/10.4102/aveh. v69i2.129

10. Jafer K, Abonesh G. Prevalence of refractive error and visual impairment among rural school-aged children of Goro district Gurage zone Ethiopia. Ethiop J Health Sci. 2010;20:353-358.

11. Kawuma M, Majeku R. A survey of refractive errors among children in lower primary schools in Kampala district. Afr Health Sci. 2002;2:69-72.

12. Naidoo KS, Raghunandan A, Mashige KP, et al. Refractive error and visual impairment in African children in South Africa. Invest Ophthalmol Vis Sci. 2003;44:3764-3770. https://doi.org/10.1167/iovs.03-0283

13. Balarabe AH, Adamu I, Abubarkar A. Vision screening to detect refractive errors in three selected schools in Birnin Kebbi, North West, Nigeria. Sahel Med J. 2015;18:61-65. https://doi.org/10.4103/1118-8561.160799

14. Kumah BD, Ebri A, Abdul-Kabir M, et al. Refractive error and visual impairment in private school children in Ghana. Optom Vis Sci. 2013;90:1456-1461. https://doi. org/10.1097/OPX.0000000000000099

15. He $M$, Huang $W$, Zheng $Y$, et al. Refractive error and visual impairment in schoo children in rural southern China. Ophthalmology. 2007:114:374-82. https://doi. org/10.1016/j.ophtha.2006.08.020

16. Sapkota YD, Adhikari BN, Pokharel GP, et al. The prevalence of visual impairment in school children of upper-middle socioeconomic status in Kathmandu. Ophthalmol Epidemiol. 2008;15:17-23. https://doi.org/10.1080/ 09286580701772011

17. Goss D, Grosvenor T, Keller JT, et al. Optometric clinical practice guideline care of patient with myopia: Reference guide for clinicians. St. Louis, MO: American Optometric Association; 2006.

18. Cooper E, Jamal N. Current status on the development and treatment of myopia. Optometry. 2012;83:179-199.

19. Morgan IG, Ohno MK, Saw SM. Myopia. Lancet. 2012;379:1739-1749. https://doi. org/10.1016/S0140-6736(12)60272-4

20. Myrowitz EH. Pediatric ophthalmology update: Juvenile myopia progression, risk factors and interventions. Saudi J Ophthalmol. 2012;26:293-297. https://doi. org/10.1016/j.sjopt.2011.03.002

21. Abdull MM, Sivasubramaniam S, Murthy GV, et al. Causes of blindness and visual impairment in Nigeria: The Nigeria National Blindness and Visual Impairment survey. Invest Ophthalmol Vis Sci. 2009;50:4115-4119. https://doi.org/10.1167/ iovs.09-3507

22. Nigerian Optometric Association (NOA). NOA report on the strategic action plan for vision 2020: The right to sight for Nigeria. Abuja: Nigerian Optometric Association; 2007-2011.

23. Faderin MA, Ajaiyeoba Al. Refractive errors in primary school children in Nigeria. Niger J Ophthalmol. 2001;9:10-14. https://doi.org/10.4314/njo.v9i1.11913

24. National Population Commission: 2006 Federal Republic of Nigeria Population and Housing Census: Distribution by sex, state, local government area and senatorial districts. Priority table volume III [homepage on the Internet]. c2010 [cited 2015 Jun 06]. Available from: file:///C:/Users/Intel/Download/Vol\%20 03\%20Table \%20DSx\%20LGAPop \%20SDistrict-PDF.pdf

25. Declaration of Helsinki. World Medical Association [homepage on the Internet] 2013 [cited 2015 May 20]. Available from: http://www.wma.net/en/ 30publications/10policies/b3/index.html

26. World Health Organization/National Eye Institute. Assessment of prevalence of visual impairment attributable to refractive error or other causes in schoo children protocol and manual of procedures [homepage on the Internet]. 2007. [cited 2015 Apr 15]. Available from: http://www.who.int/blindness/causes/ RESCProtocol.pdf

27. Aldebasi YH. Prevalence of correctable visual impairment in primary schoo children in Qassim Province, Saudi Arabia. J Optom. 2014;7:168-176. https://doi. org/10.1016/j.optom.2014.02.001

28. Yip VC, Pan CW, Lin XY, et al. The relationship between growth spurts and myopia in Singapore children. Invest Ophthalmol Vis Sci. 2012;53:7961-7966. https://doi. org/10.1167/iovs.12-10402

29. Parssinen O, Lyyra AL. Myopia and myopic progression among schoolchildren A three-year follow-up study. Invest Ophthalmol Vis Sci. 1993;34:2794-2802.

30. Goss DA. Variables related to rate of childhood myopia progression. Optom Vis Sci. 1990;67:631-636. https://doi.org/10.1097/00006324-199008000-00014

31. El-Bayoumy BM, Saad A, Choudhury AH. Prevalence of refractive error and low vision among schoolchildren in Cairo. East Mediterr Health J. 2007;13:575-579. 